\title{
Embodiment und Wirkfaktoren in Therapie, Beratung und Coaching
}

\author{
Wolfgang Tschacher · Bettina Bannwart
}

Eingegangen: 12. Oktober 2020 / Angenommen: 10. November 2020 / Online publiziert: 26. Januar 2021 (C) Der/die Autor(en) 2021

Zusammenfassung Die Kognitionswissenschaften betonen die Zusammenhänge und Wechselwirkungen zwischen geistigen und körperlichen Prozessen und deren Einbettung in die soziale und physikalische Umwelt (,Embodiment“). Dieser Ansatz erbringt Implikationen für das Verständnis von sozialer Interaktion, die durch körperliche Synchronie und allgemeine Wirkfaktoren gekennzeichnet ist. Auf Basis der Forschung zeigt sich, dass Psychotherapie, Beratung, Coaching und LehrLern-Prozesse als Tätigkeiten im Bereich des sozialen Lernens in analoger Weise mit der Verkörperung des Geistes und den therapeutischen Wirkfaktoren in Beziehung stehen. Die Therapeutenfaktoren von resilienter Abstinenz und Achtsamkeit kennzeichnen auch das lösungsorientierte Coaching.

Schlüsselwörter Embodiment · Therapeutenfaktoren · Wirkfaktoren

\footnotetext{
W. Tschacher $(\bowtie)$

Universitätsklinik für Psychiatrie und Psychotherapie, Bolligenstr. 111, 3060 Bern, Schweiz

E-Mail: wolfgang.tschacher@upd.unibe.ch

B. Bannwart $(\bowtie)$

Berufsfachschule (BFF), Monbijoustr. 21, 3011 Bern, Schweiz

E-Mail: bettinabannwart@bluewin.ch
} 


\section{Embodiment and common change factors in therapy, counseling and coaching}

Abstract Cognitive science and psychology emphasize the mutual relationships between mental and bodily processes, together with their embedding in the social and physical environment (embodiment, 4E cognition). This approach generates implications for our understanding of social interaction in general, which is characterized by nonverbal synchrony and common factors of change. Based on empirical findings, we realize that psychotherapy, counseling, coaching, and teaching, all practices of social learning, present analogous links with the embodiment of the mind and with therapeutic factors. Therapist factors such as resilience, abstinence and mindfulness are instrumental also in solution-focused coaching.

Keywords Embodiment - Therapeutic ingredients · Therapist factors

\section{Einführung: Embodiment und Wirkfaktoren in therapeutischen Interaktionen}

Das Ziel dieses Beitrags ist es zunächst, neuere Entwicklungen zu grundlegenden Fragen der Psychologie und Kognitionswissenschaft vorzustellen und Ergebnisse der schulübergreifenden Psychotherapieforschung zu skizzieren. Dabei stoßen wir auf den Embodimentansatz, die Tendenz zur Synchronisierung in der sozialen Interaktion, und auf allgemeine Wirkfaktoren in Therapiekontexten. Dies wird daraufhin in Bezug zur Praxis von Therapie, Beratung/Coaching sowie Lehr-/Lernprozessen gesetzt, um Konsequenzen aus den theoretischen und empirischen Befunden für diese Praxisfelder zu ziehen.

\subsection{Embodiment}

In der Psychologie und Kognitionswissenschaft etabliert sich seit einigen Jahren zunehmend die Perspektive des „Embodiment“, also der Verkörperung und Leiblichkeit psychischer Prozesse. Diese Perspektive besagt, dass psychische nicht von körperlichen Prozessen getrennt betrachtet werden können, stattdessen müsse man berücksichtigen, dass der Geist immer im Körper eingebettet ist, während er ihn zugleich auch beeinflusst (Storch et al. 2006; Tschacher und Storch 2012). Damit wird die Wechselwirkung und Bidirektionalität zwischen Geist und Körper durch das Embodiment des Geistes in den Vordergrund gestellt. Dieser Ansatz kritisiert sowohl die einseitige Computermetapher der Kognitivisten ${ }^{1}$ (,Kognition ist nichts als die Verarbeitung von Information - bits und bytes“) als auch der Neurowissenschaftler (,Kognition ist nichts als ein Gehirnprozess“). Der Fokus liegt nun vielmehr auf der permanenten Wechselwirkung zwischen Kognition und Körper sowie beider mit ihrer Umwelt.

\footnotetext{
1 Wir verwenden in diesem Artikel durchgängig das generische Maskulinum.
} 
Abb. 1 Schematische Darstellung der ,4E Cognition “: der Geist ist verkörpert (Embodied), enaktiv (Enactive), situiert (Embedded) und erweitert (Extended)

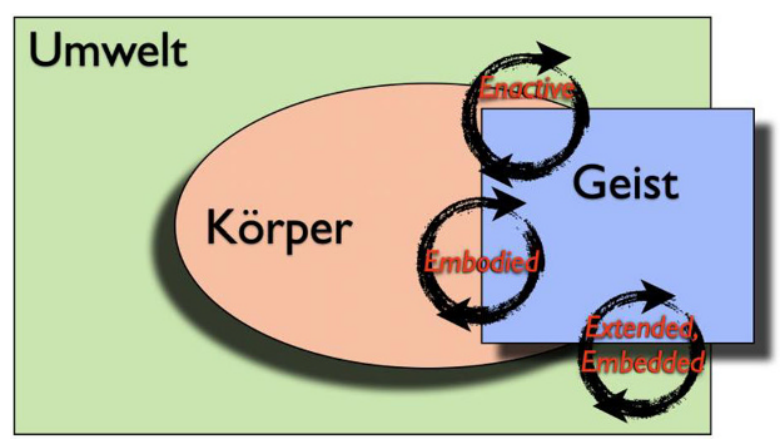

Dieses neue Verständnis wird auch als 4E Cognition bezeichnet (Abb. 1): Kognition ist zunächst verkörpert (Embodied). Kognition basiert zudem auf sensorischmotorischen Schleifen (Enactive), also nicht auf der passiven Aufnahme von Informationen, sondern der dynamischen Wechselwirkung eines Lebewesens mit seiner Umwelt. Kognition ist weiterhin situiert (Embedded), also abhängig von Kontext und aktueller Situation. Schließlich ist Kognition erweitert (Extended), wir denken demnach unter Zuhilfenahme unserer Umwelt und benutzen etwa Schreibstifte, Werkzeuge und Computer als Erweiterungen des Geistes. Kognition ist nicht auf den menschlichen Körper oder gar auf das Gehirn beschränkt und erweitert sich über die Grenzen des Körpers hinaus (Clark und Chalmers 1998). Alle vom EmbodimentAnsatz beschriebenen Zusammenhänge sind bidirektional, sie werden in Abb. 1 als Kreise dargestellt.

Die Embodiment-Perspektive hat zahlreiche Implikationen (Tschacher und Bergomi 2011). Für den Bereich der psychischen Störungen ist dies vor allem die Einsicht, dass solche Störungen immer eine körperliche Komponente besitzen. Beispielsweise zeigten Michalak et al. (2015), wie eine unbemerkte experimentelle Manipulation der Motorik beim Gehen auf einem Fließband psychische Prozesse gezielt verändern kann. Gesunde Probanden mit depressivem Gang erinnerten sich anders an vorher gelernte positive und negative Wörter als Probanden mit fröhlicher Gangart: Das übermäßige Erinnern an negative Inhalte ist ein typisches Symptom von Depressionen. Auch für Erkrankungen des Schizophreniespektrums liegen Befunde vor, die auf ein vermindertes Embodiment der Betroffenen schließen lassen (Tschacher et al. 2017), woraus neue Therapiemöglichkeiten erwachsen können (Martin et al. 2016). Die veraltet als „Geisteskrankheiten“ angesehenen Störungen betreffen damit sowohl den Geist als auch den Körper.

\subsection{Synchronie}

Eine weitere Implikation des Embodiment ist ein erweitertes Verständnis von sozialer Interaktion. Kommunikation erschöpft sich nicht im Senden und Empfangen von informationshaltigen Botschaften, sondern basiert auf psychischen und körperlichen Synchronisationen (Storch und Tschacher 2014). Das Phänomen der körperlichen und nonverbalen Synchronie wird in der Sozialpsychologie und Psychotherapiefor- 
Abb. 2 Nichtlineare Beziehung zwischen Synchronie und Affekt

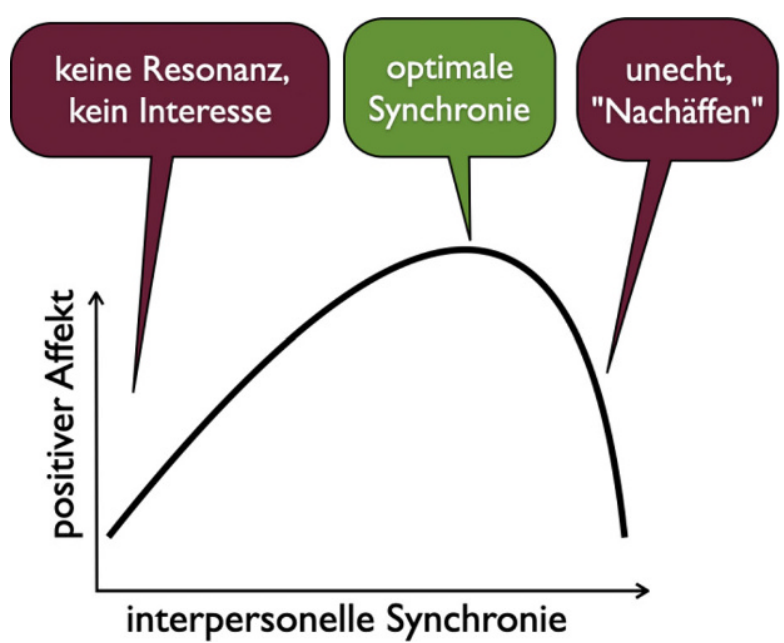

schung gegenwärtig intensiv beforscht (Wiltshire et al. 2020). Die Synchronie von Interaktionspartnern äußert sich motorisch wie auch physiologisch in einer Kopplung und Koordination von Körperbewegung, Gestik und Körperhaltung bis hin zu gemeinsamer Pulsfrequenz, Atmung und Hautleitfähigkeit (Ramseyer und Tschacher 2006; Kleinbub 2017; Tschacher und Meier 2020). Synchronie basiert auf einer sehr menschlichen Eigenart, nämlich der Tendenz, bei einer Interaktion das Gegenüber zu ,imitieren“, was aber keine willentliche Nachahmung, sondern eine automatisch sich einstellende Sozialdynamik darstellt. Häufig finden die einschlägigen Studien, dass Synchronie eine prosoziale Eigenschaft von Interaktionen ist, also in gelingender Kommunikation und bei beidseitigem höherem Engagement stärker vorhanden ist.

Grammer (1995) beschreibt die motorische Synchronisation von Paaren aus soziobiologischer Perspektive als Aspekt von Werbeverhalten. Gemäß seiner „Anatomie des Flirts“ sind gekoppelte körpersprachliche Signale ein Zeichen für Interesse. Die biologische Bedeutung der Synchronie hat allerdings auch eine Kehrseite, denn motorische Synchronie kann bewusst, in der Absicht zu täuschen, hergestellt werden. Daraus erklärt sich, dass übertriebene Synchronie rasch als nicht-authentisch wahrgenommen wird (als „Nachäffen“ oder ,,mime effect“; Boker 2004) und dann in negativen Affekt umschlagen kann. Die Beziehung zwischen Synchronie und Affekt ist damit wohl nichtlinear, umgekehrt U-förmig (Abb. 2). Solche Zusammenhänge wurden auch in Therapeut-Patient-Dyaden zwischen Synchronie und Therapieerfolg gefunden (Paulick et al. 2018; Benecke und Krause 2005).

\subsection{Wirkfaktoren}

Das Ziel der Psychotherapieforschung ist es, die Wirksamkeit verschiedener Interventionen zu erforschen. Dabei hat sich gezeigt, dass allgemeine Wirkfaktoren (common factors) wohl die wichtigste Rolle beim Therapieerfolg spielen (Grawe 1998; Wampold et al. 2018). Diese Wirkfaktoren kommen in den vielen unterschiedlichen 


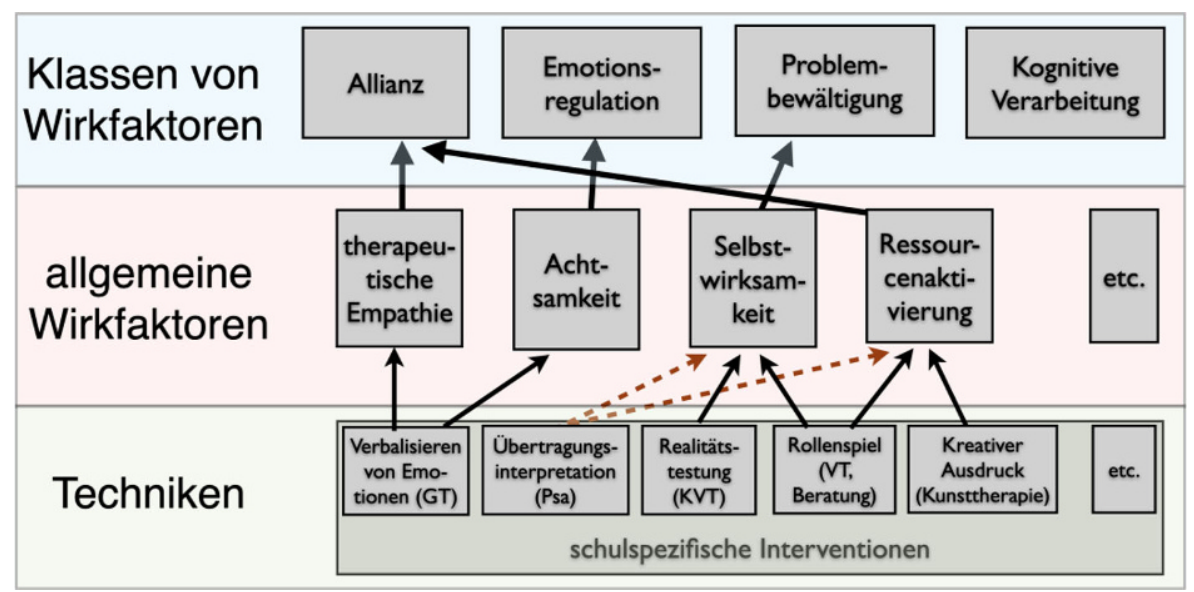

Abb. 3 Hierarchisches Modell therapeutischer Interventionen: schulspezifische Techniken $\rightarrow$ allgemeine Wirkfaktoren $\rightarrow$ übergeordnete Klassen von allgemeinen Wirkfaktoren. GT: Gesprächspsychotherapie; Psa: Psychoanalyse; KVT: kognitive Verhaltenstherapie; VT: Verhaltenstherapie. Pfeile: gefundene Zusammenhänge, gebrochene Pfeile: negative Zusammenhänge

Beratungs- und Therapieformen und bei der Behandlung verschiedenster Probleme gleichermaßen zum Tragen. Als zentraler allgemeiner Wirkfaktor gilt eine gute und vertrauensvolle therapeutische Beziehung (therapeutische Allianz). Weiterhin soll Therapie und Beratung mit den bereits vorhandenen Ressourcen des Klienten arbeiten, und diese Stärken des Klienten sollen ausgebaut werden (Ressourcenorientierung). Mehrere weitere allgemeine Wirkfaktoren wurden in der Literatur beschrieben (Pfammatter und Tschacher 2012). Entgegen den weiterhin stattfindenden Debatten zwischen den Therapieschulen scheint es inzwischen unstrittig, dass die allgemeinen Wirkfaktoren therapeutischen Erfolg besser vorhersagen als die spezifischen Therapietechniken, durch die sich Therapieschulen definieren. Dennoch sind die spezifischen Techniken dadurch nicht etwa als überflüssig und wirkungslos entlarvt, denn nur durch Techniken können allgemeine Wirkfaktoren in Gang gesetzt werden (Pfammatter und Tschacher 2016). Die Schulen scheinen den Therapieerfolg einfach auf unterschiedlichen Wegen zu erreichen, was durch ein hierarchisches Modell therapeutischer Faktoren veranschaulicht werden kann (Abb. 3).

\subsection{Therapeutenfaktoren}

Erst relativ spät wurden in der Psychotherapieforschung neben den schul- und störungsspezifischen Faktoren auch therapeutenspezifische Faktoren erforscht (Lutz und Barkham 2015). In großen Datensätzen, wie sie heute durch breite Qualitätssicherungsstudien verfügbar sind, erwiesen sich einige Therapeuten durchgängig als erfolgreicher, ohne dass dies durch das Alter, die Berufserfahrung, das Geschlecht des Therapeuten oder die Übereinstimmung des Geschlechts von Therapeut und Klient erklärt werden konnte.

Warum sind also einige Therapeuten effektiver? Man fand zum einen wiederholt, dass das Wohlbefinden des Therapeuten selbst einen ausgeprägteren Therapieerfolg 
vorhersagt und dass umgekehrt professioneller Burnout auf Seite des Therapeuten schlechtere Therapieergebnisse nach sich zieht. Pereira et al. (2017) zeigten darüber hinaus, dass Resilienz und Achtsamkeit in der Praxis wichtige Therapeuteneigenschaften sind. Resilienz ist ein Persönlichkeitszug, der eine Person unter Stress belastbar macht, was besonders in der Arbeit mit traumatisierten Klienten bedeutsam sein kann. Achtsamkeit ist definiert als eine Haltung nicht-wertender Aufmerksamkeit gegenüber inneren und äußeren Reizen. Achtsamkeit bedeutet auch, nicht unmittelbar auf Erfahrenes zu reagieren (Michalak et al. 2012). Zusammengenommen erlauben Resilienz und Achtsamkeit einem Therapeuten, auch angesichts widriger Umstände und bei der Arbeit mit schwierigen Klienten und Themen das eigene Wohlbefinden zu bewahren.

Aus einer systemtheoretischen Modellierung von therapeutischen und beraterischen Prozessen entsteht ein ähnliches Bild auf der Basis von formalen mathematischen Überlegungen (Tschacher und Haken 2019). Ausgehend von den Befunden der Synchronieforschung, die wir oben darstellten, entwickelt sich gemäß diesem Modell in therapeutischen Interaktionen eine Kopplung zwischen Therapeut und Klient. Die je eigenen Dynamiken beider Personen organisieren und koordinieren sich, und es bildet sich eine gemeinsame Dynamik heraus. Die Frage ist hierbei nun, wie ein Therapeut die gemeinsame Dynamik optimal beeinflussen kann. Es zeigt sich dabei, in systemtheoretischer Begrifflichkeit, dass ein effektiver Therapeut durch lange Relaxationszeiten, Abschirmung gegen Zufallseinflüsse von außen sowie durch hohe Stabilität ausgezeichnet sein sollte. Unter diesen Umständen wird wegen des Versklavungsprinzips der Synergetik (Haken 1990) die Selbstorganisation der Interaktion zweier Subsysteme (Therapeut und Klient) nach den Vorgaben eines der Subsysteme (des Therapeuten) ausgebildet. Tschacher und Haken (2019) bezeichneten diese Eigenschaften als die „Archimedische Funktion“ therapeutischer Einflussnahme: Der antike Wissenschaftler Archimedes entdeckte das Hebelprinzip, und der Therapeut kann als der ruhende Pol des Therapieprozesses angesehen werden. Diese theoretische Modellierung ist konsistent mit der empirischen Befundlage zu Therapeutenfaktoren: Lange Relaxationszeit und stabile Dynamik bedeuten Resilienz, Abschirmung gegen übermäßige Beeinflussung von außen ist kompatibel mit einer achtsamen und abstinenten Haltung des Therapeuten.

\section{Implikationen und Anwendungen im Coaching und im Lehr-Lern- Kontext}

\subsection{Embodiment}

Auch wenn beim Coaching das Gespräch im Zentrum steht, fließen die Erkenntnisse aus der Forschung bezüglich Embodiment in den verbalen Austausch ein. Hier ist die Situation dieselbe wie in den verhaltenstherapeutischen und psychodynamischen Therapieschulen, die zwar gemäß ihrer Theorie den Fokus auf kognitive Prozesse legen, wobei aber die Empirie zeigt, dass die körpersprachliche Ebene in der konkreten Therapiesitzung bedeutsam ist. Körperorientierte Techniken sind dagegen traditionell Bestandteil humanistischer und systemischer Therapieschulen, etwa in Form der 
Stuhltechnik in der Gestalttherapie oder der Aufstellungsarbeit in der Familientherapie (Kleve 2011). Auf systemische Hintergrundtheorien bezieht sich insbesondere die lösungsorientierte Beratung (Bamberger 2010). Es wird als besonders wichtig angesehen, mit welcher Haltung der Coach seine Arbeit tut: Einerseits geht es dabei um die Haltung im Sinne innerer Grundeinstellung, andererseits aber auch um die Haltung als Körperhaltung. Somit ist ein Bewusstsein für und das Wissen über Embodiment für Coaches von besonderer Wichtigkeit.

Bewusstes Wahr- und Einnehmen der eigenen Körperhaltung des Coachs und derjenigen des Klienten können dazu beitragen, Veränderungsprozesse in Gang zu bringen. Wenn das Problem den Coach oder den Klienten ,niederzuziehen“ droht oder wenn der Geist ,festsitzt“, kann es hilfreich sein, die Körperhaltung zu variieren, sich z. B. anders, aufrechter hinzusetzen, den Körper zu dehnen, die Schultern zu entspannen, den Nacken zu strecken. Selbst in Belastungssituationen oder bei Blockaden ist eine Person nämlich immer noch in der Lage, die Körperhaltung und Muskelspannung zu verändern und damit aktiv Einfluss auf Stimmung und Gefühle zu nehmen. Der Coach kann seinen eigenen Körper als hilfreiches Instrument im Coaching-Gespräch einsetzen: Die Positur und Körperhaltung überträgt sich in der Regel unbewusst auf den Klienten, wie die Synchronieforschung zeigt (Wechselwirkung „Enactive“ in der 4E Cognition; s. Abb. 1). Dies kann sich wiederum positiv auf die affektive Befindlichkeit des Klienten und somit auf seine Bereitschaft und Fähigkeit, Lösungen für Probleme zu finden, auswirken.

Ziele spielen im Coaching eine besondere Rolle: Was möchte der Klient verändern und welche Schritte möchte er als nächstes in diese Richtung tun? Im Zürcher Ressourcenmodell (ZRM, Storch und Krause 2002) wird mit Motto-Zielen gearbeitet, also mit metaphorisch, symbolisch und ikonisch codierten Zielen, die aufgrund ihrer starken Bildhaftigkeit unbewusste und körpernahe Ebenen des psychischen Systems ansprechen (Tschacher et al. 2014). Entsprechend dem Embodiment-Ansatz unterstützt der Körper den Geist bei der Zielformulierung: Mit körperbasierten Interventionen können Ziele entwickelt, geschärft und verankert werden. Ein Beispiel hierfür sind die die Selbstwirksamkeit fördernden Körperposituren der ,,power poses“ (Carney et al. 2010). Bidirektional gewendet, geben Körperrückmeldungen und das Wahrnehmen von somatischen Markern Auskunft darüber, ob eine Lösungsperspektive aus Sicht des Klienten passend erscheint, was im ZRM-basierten Coaching ausführlich berücksichtigt wird. Diese Implikationen des EmbodimentAnsatzes betreffen also die Wechselwirkung „Embodied“ (s. Abb. 1).

Wenn der Coach den Körper des Klienten als Ressource erkennt, die Körpersprache bzw. die Körpersignale im Coaching-Gespräch beobachtet, erhält er wichtige Hinweise auf die Befindlichkeit des Klienten - es kann gemeinsam herausgearbeitet werden, welche Körperhaltungen mit negativen oder positiven Gefühlen verknüpft sind. Durch das Einnehmen einer Körperhaltung, die mit positivem Affekt gekoppelt ist, kann bereits ein erster Schritt hin zu einer Veränderung Richtung Ziel ausgelöst werden. Der Klient lernt, Signale seines Körpers achtsam wahrzunehmen und umgekehrt seinen Körper als unmittelbare Kraftquelle zu erleben. Auf die gleiche Weise kann es dem Coach gelingen, Selbstsorge auszuüben: Er kann eigene Stimmungen und Gefühle in eine positive Richtung bewegen und gleichzeitig den Klienten damit ,anstecken“. Interessant ist auch, dass besonders wirksames Coaching oft nicht 
am Tisch oder im Sessel, sondern unterwegs, beim gemeinsamen Tun, also in Bewegung, stattfindet. In festgefahrenen Situationen kann sich ein Coaching-Gespräch beim gemeinsamen Spaziergang entlang des Flusses als förderlich erweisen (situierte Kognition: „Extended“; s. Abb. 1).

Im pädagogischen Kontext geht es wie beim Coaching gleichermaßen um LehrLern-Prozesse, und hier wird der Embodiment-Ansatz zunehmend als bedeutsam erkannt. Eine Pionierrolle hatte diesbezüglich das Projekt „Lernen in Bewegung“ des Schweizer Lehrers Eduard Buser (https://lerneninbewegung.ch/home). Bewegung und rhythmisierte Bewegung der Schüler in der Unterrichtsstunde werden beim Lernen in Bewegung nicht mehr als störend sanktioniert, sondern zum Instrument der Stoffvermittlung. Ganz im Sinne des Embodiment-Ansatzes wird das Bildungspotenzial von Bewegung genutzt (Zimmer 2020).

Wenn man berücksichtigt, dass nicht nur der Geist den Körper, sondern auch der Körper den Geist und die Emotion beeinflusst, ist der Einstieg in den Unterricht mit einer Form von Körperarbeit naheliegend. Auch ohne formale Anleitung oder Übungen überträgt sich die Körperhaltung der Lehrperson auf die Lernenden. Das aufrechte Stehen mit geradem Rückgrat wirkt sich unmittelbar positiv auf das Selbstbild aus, man fühlt sich stärker, kompetenter, mutiger. Anschließende Streckund Dehnübungen lassen den ganzen Körper spüren, erlauben es, Gleichgewicht und Stabilität zu finden und durch An- und Entspannung in einen angenehmen Tonus zu kommen. Es bestehen positive Erfahrungen in der Erwachsenenpädagogik mit vor den Unterricht eingefügten TaiChi-Sequenzen. Die langsamen und fließenden Bewegungen begünstigen die Synchronisation der Gruppe. Lernende wie Lehrpersonen fühlen sich nach einer Anfangssequenz mit einfachen Übungen aus TaiChi und QiGong erfrischt und leistungsbereiter. Körperübungen haben offensichtlich einen spür- und sichtbaren Effekt auf die Stimmung. Wenn man zudem beachtet, dass positive Emotionen wesentlich sind für Intelligenz und Kreativität und eine direkte Wirkung auf die Lernbereitschaft und -fähigkeit haben, erscheint der Einbezug des Körpers doppelt sinnvoll.

\subsection{Wirkfaktoren im Coaching}

Ein wichtiger theoretischer Ansatz in Coaching und Beratung ist die Lösungsorientierung nach Steve de Shazer und Insoo Kim Berg (de Shazer 1992; de Jong und Berg 2008). Der lösungsorientierte Ansatz legt in Beratung und Coaching den Fokus nicht auf das Problem und die Problemursachen, sondern im Sinne einer salutogenetischen Haltung auf die Entwicklung einer möglichen Lösung. Hier bestehen große Parallelen zu Grawes (1998) Ressourcenaktivierung als allgemeinem Wirkfaktor der Psychotherapie (s. Abb. 3). Grundsätzlich wird davon ausgegangen, dass es immer eine Lösung (manchmal auch mehrere) für ein Problem gibt und dass der Klient, als der alleinige Experte für sein Leben, fähig ist, eigene Lösungsideen zu entwickeln.

Die Lösungsorientierung basiert auf konstruktivistischen und sozialkonstruktivistischen Ansichten, wie sie in systemischen Therapierichtungen vorherrschen (Schweitzer und v. Schlippe 2007). Die konstruktivistische Orientierung besagt in der Anwendung, dass jeder in seiner eigenen, nur ihm zugänglichen Lebenswelt lebt und dass eine objektive Wahrnehmung dieser Lebenswelt von außen, also durch 
einen Therapeuten oder Coach, nicht möglich ist. Zielgerichtete, unmandatierte Beeinflussungen der Konstruktionen des Gegenübers werden demzufolge a priori ausgeschlossen (Pfister-Wiederkehr 2019). Daraus folgt, dass ein lösungsorientierter Coach eine achtsame Grundeinstellung mit nicht-wertender Aufmerksamkeit einnimmt. Sein Ziel ist, dass der Klient seine eigene Lösungsvision (er)findet. Der Coach ist lediglich Experte für den Prozess der Lösungskonstruktion, bleibt aber in einer klaren Distanz zur inhaltlichen Ebene des Problems. Er ist dem Klienten durch geschicktes und achtsames Befragen behilflich, seine eigenen Ziele präzise $\mathrm{zu}$ formulieren, seine Ressourcen und Kompetenzen zu entdecken und zu aktivieren und kleine erste Veränderungen (die zu großen führen können) zu lancieren. Die Interventionstechnik ist damit eine Form von nichtwertender Abstinenz, wodurch beim Klienten allgemeine Wirkfaktoren wie Ressourcenaktivierung und erhöhte Selbstwirksamkeit (der Klient als aktiver Gestalter seines Lebens) angestoßen werden sollen.

Hier zeigt sich ein Bezug auch zu den theoretisch entwickelten Therapeuteneigenschaften (Tschacher und Haken 2019). Der lösungsorientierte Coach arbeitet langsam, er will nicht zu schnell verstehen - und gibt stattdessen dem Klienten Zeit und Raum, seine Gedanken in Ruhe zu sortieren und sein Ziel selbst zu entwickeln. Dazu gehört auch eine Haltung des achtsamen Nichtsofortreagierens des Coachs, da die Lösungsorientierung keine Bewertung der durch den Klienten erzeugten Lösungen durch den Coach vorsieht.

\section{Diskussion}

In diesem Beitrag haben wir uns auf die neueren Entwicklungen in der Psychologie und Kognitionswissenschaft bezogen, die wechselseitige Zusammenhänge zwischen geistigen und körperlichen Prozessen betonen und die Einbettung von Geist/ Kognition und Körper in die soziale und physikalische Umwelt ansprechen. Dieser Embodiment-Ansatz hat weitreichende Implikationen für das Verständnis therapeutischer Interaktionen, wie er etwa in der Synchronieforschung zum Tragen kommen. Weiterhin haben wir den gegenwärtigen Stand der Wirkfaktorenforschung in der Psychotherapie skizziert. Im zweiten Teil war es unser Ziel, diese Erkenntnisse für den Kontext von Beratung, Coaching und Pädagogik aufzubereiten und nutzbar zu machen.

Wir machten hierbei die folgende Annahme: Die genannten Praktiken, also Psychotherapie, Beratung, Coaching und Lehr-Lern-Prozesse, sind nicht etwa kategorial unterschiedlich, wie der geringe Austausch zwischen den beteiligten Disziplinen vermuten lassen könnte. Es handelt sich stets um Tätigkeiten im Bereich des sozialen Lernens. Folglich sollten die Befunde zum Embodiment des Geistes und zu Wirkfaktoren für alle diese Praktiken in analoger Weise anwendbar sein. Tatsächlich finden sich solche Analogien und konsistenten Anwendungen. So können Methoden der lösungsorientierten Beratung durchaus in Einklang mit der psychotherapeutischen Wirkfaktorenforschung gesehen werden. Die aus der Systemtheorie und der empirischen Forschung abgeleiteten Therapeutenfaktoren von resilienter Abstinenz und Achtsamkeit des Therapeuten findet man ebenfalls bei lösungsorientierten Coaches, 
wenn auch aus unterschiedlichen Hintergrundtheorien abgeleitet. Mehrere Folgerungen aus der Embodiment-Forschung lassen sich zudem auch im Coaching und in Lehr-Lern-Prozessen sinnvoll einsetzen.

Einschränkend kann man hinzufügen, dass die gefundenen Analogien nicht bedeuten, dass man den postmodernen Konstruktivismus der Lösungsorientierung unkritisch übernehmen sollte. Die Ergebnisse empirischer Forschung und ihre gelingende Generalisierung sind unserer Meinung nach nicht nur ein Resultat von sozialer Konstruktion und Erfindung. Weiterhin scheint es uns nicht zwingend, im Coaching auf die Problemanalyse zu verzichten. Auch Psychopathologie und allgemeine psychologische und soziale Probleme sind verkörpert, also ein legitimer Teil der Embodiment-Forschung. Wenn sich daraus neue Interventionsmöglichkeiten und Beratungsansätze entwickeln sollten, wäre das trotz einer salutogenetischen Ausrichtung zu begrüßen.

Funding Open access funding provided by University of Bern

Open Access Dieser Artikel wird unter der Creative Commons Namensnennung 4.0 International Lizenz veröffentlicht, welche die Nutzung, Vervielfältigung, Bearbeitung, Verbreitung und Wiedergabe in jeglichem Medium und Format erlaubt, sofern Sie den/die ursprünglichen Autor(en) und die Quelle ordnungsgemäß nennen, einen Link zur Creative Commons Lizenz beifügen und angeben, ob Änderungen vorgenommen wurden.

Die in diesem Artikel enthaltenen Bilder und sonstiges Drittmaterial unterliegen ebenfalls der genannten Creative Commons Lizenz, sofern sich aus der Abbildungslegende nichts anderes ergibt. Sofern das betreffende Material nicht unter der genannten Creative Commons Lizenz steht und die betreffende Handlung nicht nach gesetzlichen Vorschriften erlaubt ist, ist für die oben aufgeführten Weiterverwendungen des Materials die Einwilligung des jeweiligen Rechteinhabers einzuholen.

Weitere Details zur Lizenz entnehmen Sie bitte der Lizenzinformation auf http://creativecommons.org/ licenses/by/4.0/deed.de.

\section{Literatur}

Bamberger, G. C. (2010). Lösungsorientierte Beratung (4. Aufl.). Weinheim, Basel: Beltz.

Benecke, C., \& Krause, R. (2005). Facial affective relationship offers of patients with panic disorder. Psychotherapy Research, 15(3), 178-187.

Boker, S. M. (2004). Context dependence of interpersonal coordination during social interaction. Vortrag an der Konferenz Learning and multimodal communication, Chicago.

Carney, D. R., Cuddy, A. J., \& Yap, A. J. (2010). Power posing: brief nonverbal displays affect neuroendocrine levels and risk tolerance. Psychological Science, 21(10), 1363-1368. https://doi.org/10.1177/ 0956797610383437.

Clark, A., \& Chalmers, D. J. (1998). The extended mind. Analysis, 58, 7-19.

Grammer, K. (1995). Signale der Liebe: die biologischen Gesetze der Partnerschaft. München: Deutscher Taschenbuch Verlag.

Grawe, K. (1998). Psychologische Therapie. Göttingen: Hogrefe.

Haken, H. (1990). Synergetik - eine Einführung. (Nichtgleichgewichts-Phasenübergänge und Selbstorganisation in Physik, Chemie und Biologie). Berlin: Springer.

De Jong, P., \& Berg, I. K. (2008). Lösungen (er)finden. Dortmund: modernes lernen.

Kleinbub, J.R. (2017). State of the art of interpersonal physiology in psychotherapy: a systematic review. Frontiers in Psychology. https://doi.org/10.3389/fpsyg.2017.02053.

Kleve, H. (2011). Aufgestellte Unterschiede: systemische Aufstellung und Tetralemma in der Sozialen Arbeit. Heidelberg: Carl-Auer. 
Lutz, W., \& Barkham, M. (2015). Therapist effects. In R. L. Cautin \& S. O. Lilienfeld (Hrsg.), The encyclopedia of clinical psychology (S. 1-6). Hoboken: Wiley.

Martin, L.A., Koch, S.C., Hirjak, D., \& Fuchs, T. (2016). Overcoming disembodiment: The effect of movement therapy on negative symptoms in schizophrenia. A multicenter randomized controlled trial. Frontiers in Psychology, 7, 483. https://doi.org/10.3389/fpsyg.2016.00483.

Michalak, J., Heidenreich, T., \& Williams, J.M. G. (2012). Achtsamkeit. Göttingen: Hogrefe.

Michalak, J., Rohde, K., \& Troje, N.F. (2015). How we walk affects what we remember: gait modifications through biofeedback change negative affective memory bias. Journal of Behavior Therapy and Experimental Psychiatry, 46, 121-125.

Paulick, J., Deisenhofer, A.-K., Ramseyer, F., Tschacher, W., Boyle, K., Rubel, J., \& Lutz, W. (2018). Nonverbal synchrony: a new approach to understand psychotherapeutic processes and drop-out. Journal of Psychotherapy Integration, 28, 367-384. https://doi.org/10.1037/int0000099.

Pereira, J.-A., Barkham, M., Kellett, S., \& Saxon, D. (2017). The role of practitioner resilience and mindfulness in effective practice: a practice-based feasibility study. Administration and Policy in Mental Health and Mental Health Services Research, 44, 691-704. https://doi.org/10.1007/s10488-0160747-0.

Pfammatter, M., \& Tschacher, W. (2012). Wirkfaktoren der Psychotherapie - eine Übersicht und Standortbestimmung. Zeitschrift für Psychiatrie, Psychologie und Psychotherapie, 60, 67-76.

Pfammatter, M., \& Tschacher, W. (2016). Klassen allgemeiner Wirkfaktoren der Psychotherapie und ihr Zusammenhang mit Therapietechniken. Zeitschrift für Klinische Psychologie und Psychotherapie, 45(1), 1-13. https://doi.org/10.1026/1616-3443/a000331.

Pfister-Wiederkehr, D. (2019). Beraten und Coachen: Lösungs- und kompetenzorientierte Bausteine für erfolgreiche Gesprächsführung, kurz und prägnant. Norderstedt: BoD Books on Demand.

Ramseyer, F., \& Tschacher, W. (2006). Synchrony: a core concept for a constructivist approach to psychotherapy. Constructivism in the Human Sciences, 11, 150-171.

Schweitzer, J., \& v. Schlippe, A. (2007). Lehrbuch der systemischen Therapie und Beratung. Göttingen: Vandenhoeck \& Ruprecht.

De Shazer, S. (1992). Der Dreh. Heidelberg: Carl-Auer.

Storch, M., \& Krause, F. (2002). Selbstmanagement-ressourcenorientiert: Grundlagen und Trainingsmanual für die Arbeit mit dem Zürcher Ressourcen Modell (ZRM). Bern: Huber.

Storch, M., \& Tschacher, W. (2014). Embodied Communication. Kommunikation beginnt im Körper, nicht im Kopf. Bern: Huber.

Storch, M., Cantieni, B., Hüther, G., \& Tschacher, W. (2006). Embodiment. Die Wechselwirkung von Körper und Psyche verstehen und nutzen. Bern: Huber.

Tschacher, W., \& Bergomi, C. (Hrsg.). (2011). The implications of embodiment: Cognition and communication. Exeter: Imprint Academic.

Tschacher, W., \& Haken, H. (2019). The process of psychotherapy: causation and chance. Cham: Springer Nature.

Tschacher, W., \& Meier, D. (2020). Physiological synchrony in psychotherapy sessions. Psychotherapy Research, 30, 558-573. https://doi.org/10.1080/10503307.2019.1612114.

Tschacher, W., \& Storch, M. (2012). Die Bedeutung von Embodiment für Psychologie und Psychotherapie. Psychotherapie in Psychiatrie, Psychotherapeutischer Medizin und Klinischer Psychologie, 17, 17-31.

Tschacher, W., Giersch, F., \& Friston, K. (2017). Embodiment and schizophrenia: a review of implications and applications. Schizophrenia Bulletin, 43, 745-753. https://doi.org/10.1093/schbul/sbw220.

Tschacher, W., Munt, M., \& Storch, M. (2014). Tanz: eine psychotherapeutische Technik? Psychotherapie in Psychiatrie, Psychotherapeutischer Medizin und Klinischer Psychologie, 19, 169-181.

Wampold, B.E., Imel, Z.E., \& Flückiger, C. (2018). Die Psychotherapie-Debatte - Was Psychotherapie wirksam macht. Bern: Hogrefe.

Wiltshire, T. J., Philipsen, J.S., Trasmundi, S.B., Jensen, T. W., \& Steffensen, S. V. (2020). Interpersonal coordination dynamics in psychotherapy: a systematic review. Cognitive Therapy and Research, 44(4), 752-773. https://doi.org/10.1007/s10608-020-10106-3.

Zimmer, R. (2020). Handbuch Bewegungserziehung. Grundlagen für Ausbildung und pädagogische Praxis. Freiburg/Br.: Herder. 


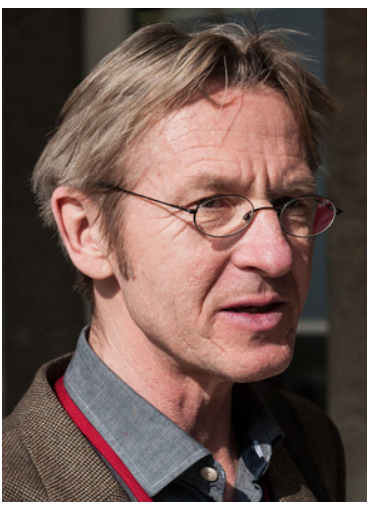

Wolfgang Tschacher Prof. Dr. phil., geb. 1956, Psychologiestudium und Dissertation Universität Tübingen, Habilitation Universität Bern, heute Abteilungsleiter Experimentelle Psychologie der Universitätsklinik für Psychiatrie und Psychotherapie Bern. Arbeitsschwerpunkte: Psychotherapieforschung, Embodiment, dynamische Systeme und Selbstorganisation, Kunstpsychologie.

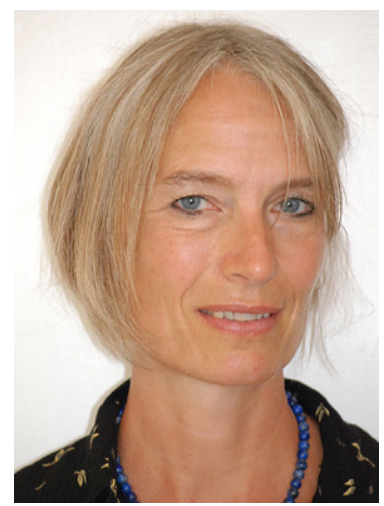

Bettina Bannwart geb. 1961, ist Pädagogin und Coach (MAS Lösungs- und Kompetenzorientierung FHZ Luzern). Als Lehrerin und Coach begleitet sie seit 2001 an einer Berufsfachschule junge Erwachsene auf dem Weg in die duale Berufsausbildung. Schwerpunkte ihrer Arbeit sind maßgeschneiderte Bildungspläne, individuelles Coaching und Beratung sowie Körperarbeit. 